\title{
A pediatric case of double gallbladder with gallstone
}

\author{
Abdullah O. Bawazir, MSc, Osama A. Bawazir, FRCSC, RCS, Razan O. Bawazir, MSc.
}

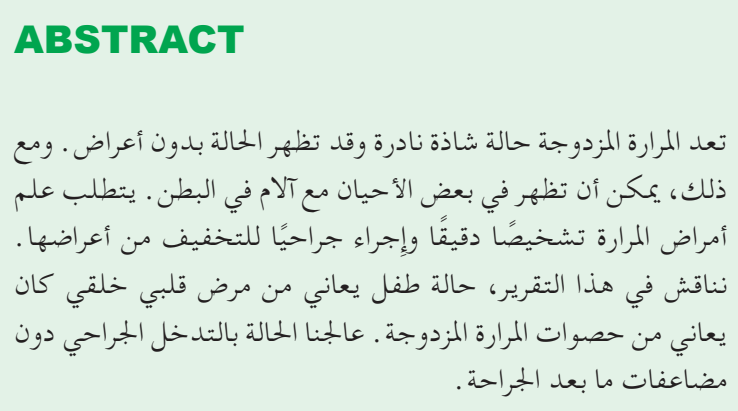

Double gallbladder is a rare anomaly and an asymptomatic condition. However, it can sometimes present with abdominal pain. Gallbladder pathology requires precise diagnosis and a surgical procedure to relieve its symptoms. Here, we discuss a case of a child suffered from a congenital heart disease who presented with double gallbladder having gallstones. The case was resolved via surgical intervention without postoperative complications.

Keywords: double gallbladder, duplicated gallbladder, duplicated gallbladder in pediatric

Saudi Med J 2020; Vol. 42 (4): 441-444 doi: 10.15537/smj.2021.42.4.20200534

From the College of Medicine (Bawazir A, Bawazir R), King Saud bin Abdulaziz University for Health Sciences, Jeddah; from the Department of Surgery (Bawazir O), Faculty of Medicine, Umm Al-Qura University, Makkah, Kingdom of Saudi Arabia.

Received 7th October 2020. Accepted 3rd January 2021.

Address correspondence and reprint request to: Dr. Abdullah O. Bawazir, College of Medicine, King Saud bin Abdulaziz University for Health Sciences, Jeddah, Kingdom of Saudi Arabia. E-mail: Oabawazir@uqu.edu.sa

ORCID ID: https://orcid.org/0000-0002-4213-7377
$\mathrm{D}$ uplication of gallbladder is considered an uncommon congenital abnormality. ${ }^{1}$ It is rarely found before any surgical operations, leading to problematic intervention and subsequently necessitating open surgical operation and complications. ${ }^{2}$ Therefore, pre-surgical diagnosis is essential to prevent any surgical or postsurgical complications. ${ }^{1}$ Although duplicated gallbladder is found in both genders, its prevalence is higher in females. ${ }^{3}$ Symptomatic conditions of duplicated gallbladder are usually associated with cholecystitis, cholangitis, gallstone disease, and pancreatitis but are rarely diagnosed with carcinoma. ${ }^{2,4}$ Surgery is considered to be the ideal treatment choice of symptomatic duplicated gallbladder. ${ }^{4}$

Case Report. Patient information. Our case report presents a 4-year-old female with congenital heart disease particularly a dextro-transposition of the great arteries with a large atrial septal defect, which have been corrected by surgery at age of 14-months.

Diagnostic assessment and clinical features. At the age of one year, she underwent an abdominal ultrasound, and a duplicated gallbladder was observed. Also, one of the gallbladders contained an echogenic concentrate (Figure 1). However, no acoustic shadowing was evident, thus, overruling the possibility of calcification. At the age of 4 years, she developed frequent pelvic pain attacks. An ultrasound suggested that the gallbladders contained 2 stones: one in the neck and one in the fundus (Figure 2). Therefore, she underwent laparoscopic cholecystectomy after availing parent's consent.

Therapeutic intervention. The patient was made to lie-down in a supine position, and general anesthesia was administered. The peritoneal cavity was accessed by open technique, by putting a 5 -mm port through infraumbilical incision. The double looking gallbladder was seen with double fundus and looked like a common wall. Then, the Calot triangle was dissected out. There were 2 complete walls with 2 separate neck and cystic ducts, which drained into the common bile duct separately. Both cystic ducts and arteries were clipped 


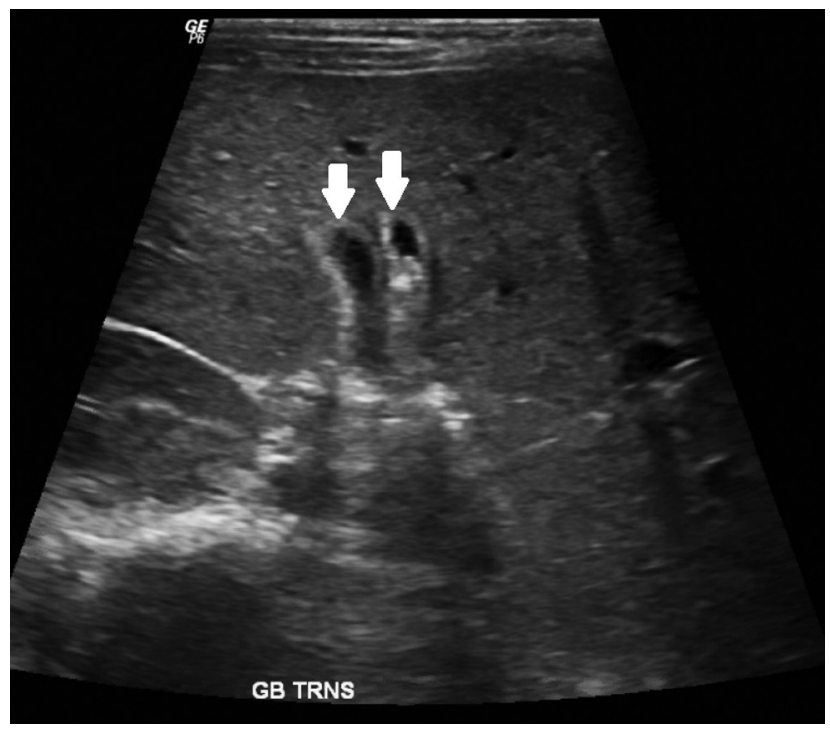

Figure 1 - Ultrasound at age of 1 year showing double gallbladder (white arrow).

with double clips and were separated in between. Subsequently, the rest of the gallbladder was dissected from its fossa off the liver with the help of Ala hook (Figure 3). All entry wounds were cleaned and stitched in double layers, with skin and subcutaneous tissue separately. Monocryl suture 5-0 was used for used for the underlying sheath and Vicryl surgical sutures 4-0 for the muscle. The patient endured the procedure properly.

Follow-up and outcomes. The patient was followed-up regularly and was doing well post-operation with no complications (Figure 4).

Discussion. Gallbladder duplication is an infrequent congenital defect, which has a prevalence of one in 4000 of postmortem samples. ${ }^{1}$ However, the precise incidence of this condition is difficult to attain due to its asymptomatic manifestation. Also, incidental encounter of radiological, surgical, and cadaveric findings are the only sources of documented cases. ${ }^{3}$ True duplicated gallbladders are usually present with 2 cystic ducts. Anatomy of several gallbladders has shown that each gallbladder has valves at its neck, tunica muscularis,

Disclosure. Authors have no conflict of interests, and the work was not supported or funded by any drug company.

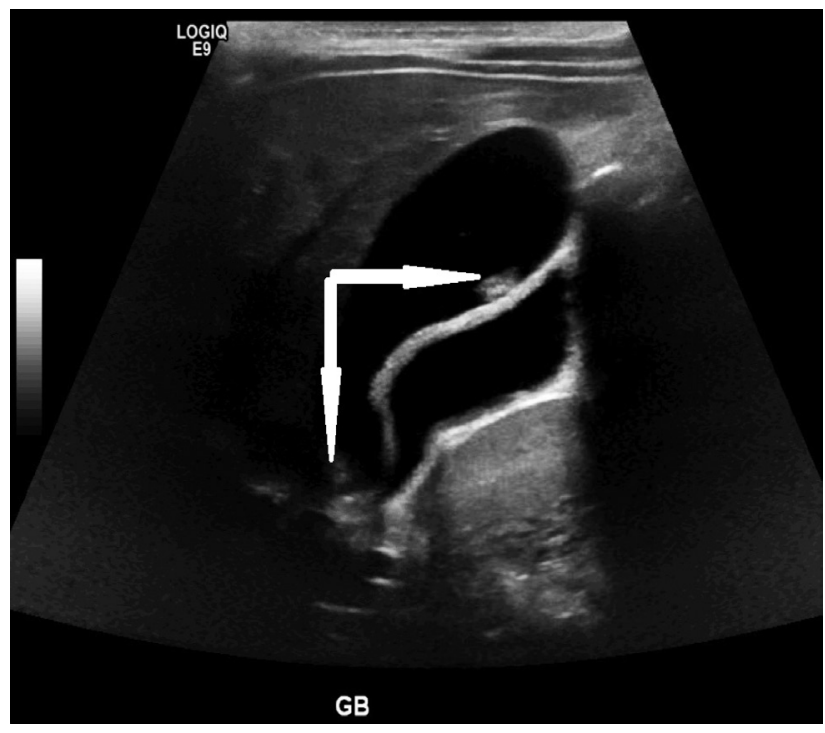

Figure 2 - Two gallstone in the superficial gallbladder, one at the neck and the other at the funds (white arrow).

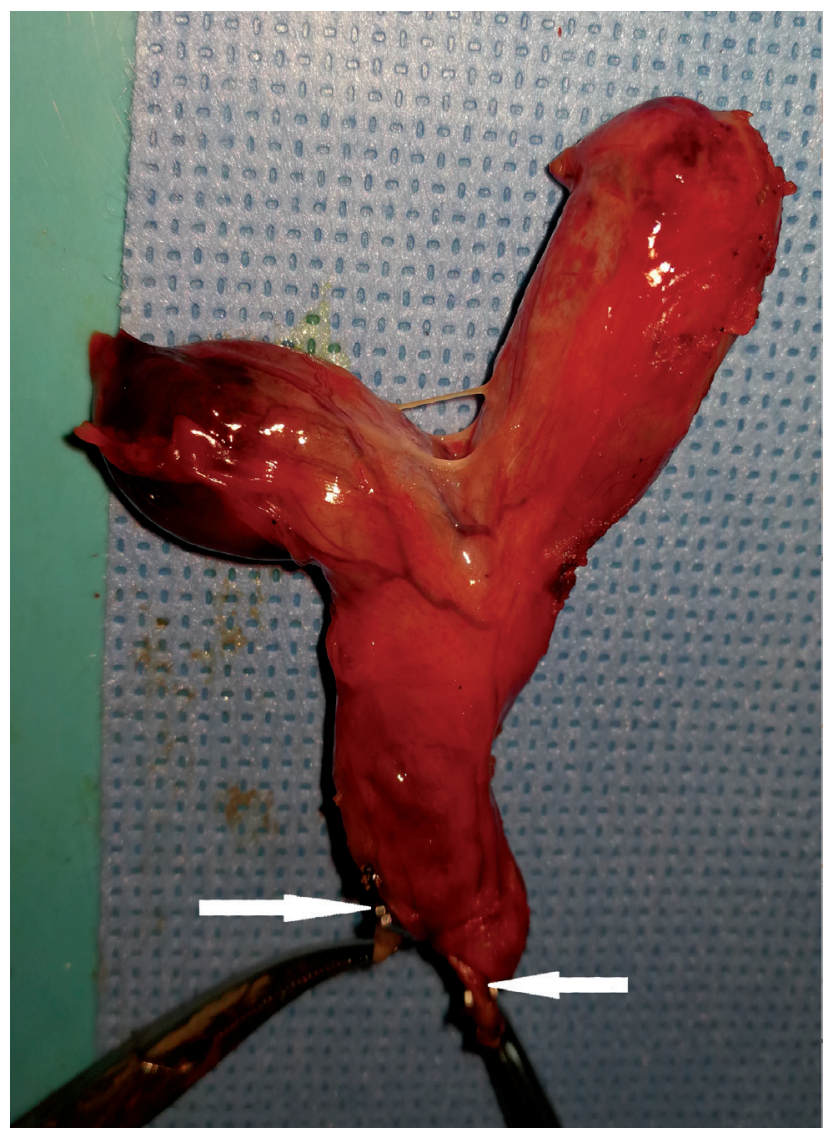

Figure 3 - Two gallstone in the superficial gallbladder, one at the neck and the other at the funds (white arrow). 


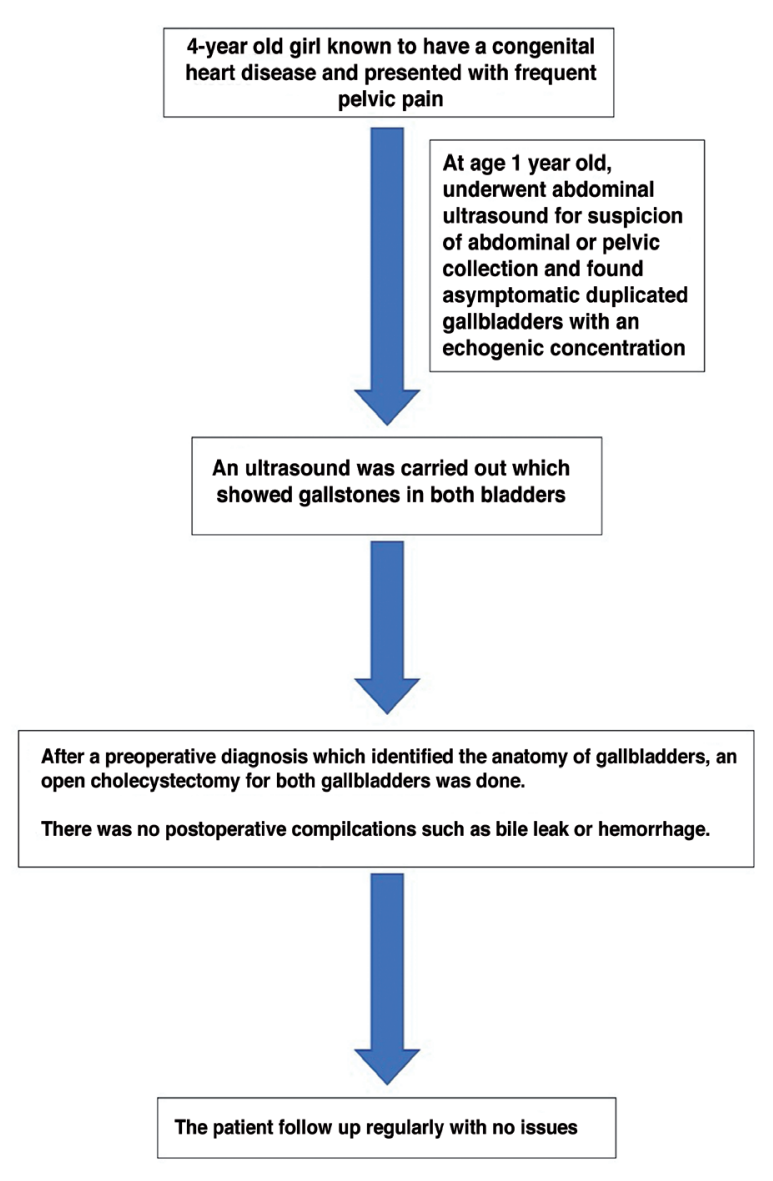

Figure 4 - Case report timeline.

and to focus bile out. ${ }^{6}$ The Harlaftis' classification is frequently used for duplicated gallbladders irregularities and is divided into 3 types. $^{7}$ In type I, the primary gallbladder contains an incompletely divided secondary gallbladder, partially split V-shaped connected in the neck only, or a completely divided Y-shaped gallbladders with the 2 cystic ducts merging into the common cystic duct. Moreover, these subtypes of type I completely sink into the common bile duct via a solitary cystic duct and are usually found beside each other in the same gallbladder fossa. In type II, the most common form is $\mathrm{H}$-type with 2 divided gallbladders emptying into the common bile duct via 2 distinct cystic ducts. It can also be trabecular type, along with any of the cystic ducts emptying toward either the right or left hepatic ducts. ${ }^{\text {? }}$ However, these anomalies occur during fetal genesis. At week 7 of fetal gestation, the hepatic diverticulum gives rise to gallbladder. An assumption suggests that type I duplications occur in later stages of development, while type II occurs as an early development error. ${ }^{8}$ Type III is an anomaly that comprises 3 gallbladders emptying through 3 different cystic ducts. ${ }^{7}$

Double gallbladders are usually found by chance or due to symptoms that are related to gallstones or inflammation of gallbladder, empyema, and carcinoma. ${ }^{1,9}$ Also, these symptoms consist of epigastric radiating pain, vomiting, and fever.' However, the formation of gallstone is the most common complication with a similar risk to that of a single gallbladder. ${ }^{1}$

A single gallbladder usually appears as hypoechoic, small rectangular, or oval cystic structure, which is found in the liver inferiorly, near the intestinal loop, and on the right side of the intrahepatic umbilical vein. Moreover, duplication of the gallbladder is considered when an extra cystic structure is observed in the gallbladder fossa. It has a specific appearance that differentiates it from other pathological conditions such as duplicated choledochal and duodenal cysts. ${ }^{10}$ Moreover, in our case study, the patient was known to have a congenital heart disease, suggesting a probability of an association between these birth anomalies. However, there have not been any reports of this suspected relatedness.

Ultrasonography is the most helpful diagnostic instrument in gallbladder evaluation due to its high sensitivity in detecting gallstones cholecystitis and anatomical anomalies. However, in cases of double gallbladders, this technique may not differentiate between pathological conditions such as folded gallbladder, and Phrygian cap. ${ }^{4}$ Furthermore, preoperative assessment is advised because it helps surgeons to be informed of any anatomical variations and reduce complications of biliary or vascular injuries. ${ }^{4}$ However, if a preoperative diagnosis such as magnetic resonance cholangiopancreatography is not carried out, an interoperative cholangiogram can help in identifying structural anomalies of gallbladder during laparoscopic cholecystectomy. ${ }^{1}$

Differential diagnosis of duplicated gallbladder might include hepatic cysts and persistent right umbilical vein. A hepatic cyst is usually found on the right hepatic lobe anterior segment, and the persistent right umbilical vein rests right to the gallbladder, and might be associated with many malformations. ${ }^{10}$ However, duplicated gallbladders are not associated with lethal anomalies, except for foregut malformations. ${ }^{10}$

In cases of symptomatic duplicated gallbladder, surgery is advised. However, prophylactic cholecystectomy should not be considered for asymptomatic patients. ${ }^{3}$ 
In case of symptomatic patients, it is recommended to get rid of both the gallbladders so as to prevent the recurrence of the disease on the other bladder later in life. ${ }^{1}$ In the past, an open cholecystectomy was recommended for identification and management of duplicated gallbladders pathology. Nowadays, with the help of the new imaging modalities, a preoperative diagnosis can be made and is followed by a laparoscopic procedure, having low morbidity rates. ${ }^{3}$ Duplicated gallbladder are rare, without any increased risk of biliary leakage or cancer of the gallbladder. Also, it is unrelated to other biliary anomalies. ${ }^{1}$

In conclusion, duplicated gallbladder is a rare condition that is incidentally evident. It is mostly asymptomatic. However, if the symptoms persist, it can be related to single gallbladder pathological conditions such as cholelithiasis or cholecystitis, along with jaundice, vomiting, and fever. An accurate preoperative or intraoperative diagnosis is required to recognize gallbladder anatomical variations and prevent further complication postoperatively. Complete laparoscopic resection for symptomatic duplicated gallbladders is safe and prevents its recurrence.

Acknowledgment. We would like to express my deep gratitude to Dr. Zergham Zia for his valuable support on radiology image in this article. Lastly, we gratefully acknowledge Manuscript Edit (www.manuscriptedit.com) for English language editing.

\section{References}

1. Al Rawahi A, Al Azri Y, Al Jabri S, Alfadli A, Al Aghbari S. Successful laparoscopic management of duplicate gallbladder: A case report and review of literature. Int J Surg Case Rep 2016; 21: 142-146.

2. Musleh MG, Burnett H, Rajashanker B, Ammori BJ. Laparoscopic double cholecystectomy for duplicated gallbladder: A case report. Int J Surg Case Rep 2017; 41: 502-504.

3. Vezakis A, Pantiora E, Giannoulopoulos D, Fontara S, Kontis E, Polydorou A, et al. A duplicated gallbladder in a patient presenting with acute cholangitis. A case study and a literature review. Ann Hepatol 2019; 18: 240-245.

4. Apolo Romero EX, Gálvez Salazar PF, Estrada Chandi JA, González Andrade F, Molina Proaño GA, Mesías Andrade FC, et al. Gallbladder duplication and cholecystitis. J Surg Case Rep 2018; 2018: rjy158.

5. Girish ML, Keshav MM, Raghunath BV, Sunil B. Gallbladder duplication associated with duodenal atresia. J Neonatal Surg 2013; 2: 46.

6. Causey MW, Miller S, Fernelius CA, Burgess JR, Brown TA, Newton C. Gallbladder duplication: evaluation, treatment, and classification. J Pediatr Surg 2010; 45: 443-446.

7. Zhou DK, Huang Y, Kong Y, Ye Z, Ying LX, Wang WL. Complete laparoscopic cholecystectomy for a duplicated gallbladder: A case report. Medicine (Baltimore) 2020; 99: e18363.

8. Diaz-Miron JL, Overman RE, Kunisaki SM. Laparoscopic double cholecystectomy for biliary dyskinesia in a child with duplicated gallbladders. J Pediatr Surg Case Rep 2019; 42: 1-3.

9. Kim RD, Zendejas I, Velopulos C, Fujita S, Magliocca JF, Kayler LK, et al. Duplicate gallbladder arising from the left hepatic duct: report of a case. Surg Today 2009; 39: 536-539.

10. Sifakis S, Mantas N, Koumantakis G, Koukoura O. Prenatal diagnosis of gallbladder duplication. Ultrasound Obstet Gynecol 2007; 30: 362-363. 